\title{
EFEITOS DE PERDA DE REFORÇADORES SOBRE O SEGUIR REGRAS EM PARTICIPANTES FLEXÍVEIS E INFLEXÍVEIS
}

\author{
EFFECTS OF LOOSING REINFORCERS ON RULE FOLLOWING IN FLEXIBLE AND \\ INFLEXIBLE PARTICIPANTS
}

\author{
Ana Rachel Pinto, Carla Cristina Paiva Paracampo e \\ Luiz Carlos de Albuquerque
}

UNIVERSIDADE FEDERAL DO PARÁ, BRASIL

\begin{abstract}
RESUMO
Com o objetivo de verificar se participantes inflexíveis apresentam maior probabilidade de seguir regras do que participantes flexíveis, quando o seguir regras produz perda de reforçadores, onze estudantes universitários, previamente classificados de flexíveis $(\mathrm{n}=6)$ e de inflexíveis $(\mathrm{n}=5)$, foram expostos a um procedimento informatizado de escolha de acordo com o modelo; a tarefa era apontar cada um dos três estímulos de comparação em uma dada sequência. As sequências corretas evitavam e as incorretas produziam a perda de reforçadores. $\mathrm{O}$ experimento consistia de três fases. A Fase 1 era iniciada com uma instrução mínima, a Fase 2, com uma instrução correspondente às contingências e a Fase 3, com uma instrução discrepante das contingências. Na Fase 1 (linha de base), nenhuma sequência era punida. O seguimento de instrução evitava a perda de reforçadores na Fase 2 e produzia a perda de reforçadores na Fase 3. Todos os 11 participantes seguiram a instrução na Fase 2 e 10 ( 5 flexíveis e 5 inflexíveis) dos 11 participantes deixaram seguir a instrução na Fase 3. Os resultados sugerem que o seguimento de instruçôes tende a ser abandonado quando produz perda de reforçadores, independentemente de se o participante é classificado de flexível ou de inflexível.
\end{abstract}

Palavras-chave: Regras e contingências, perda de reforçadores, participantes flexíveis e inflexíveis, histórias pré-experimentais

\section{ABSTRACT}

This study invetsigated whether inflexible participants have a higher probability of rule-following than flexible participants, when rule-following produces reinforcer loss. Eleven undergraduate students, previously classified as flexible $(\mathrm{N}=6)$ and inflexible $(\mathrm{N}=5)$ were exposed to a computerized matching to sample procedure; the task was to point out each of three comparison stimuli in a given sequence. The correct sequences would avoid reinforcer loss and the incorrect ones would produce it. The experiment had three phases. Phase 1 began with minimal instructions, Phase 2 included instructions that corresponded to the contingencies applied, and Phase 3 provided instructions discrepant from contingencies. In Phase 1 (baseline), no sequence was punished. Instruction-following avoided reinforcer loss in Phase 2 and produced reinforcer loss in Phase 3. All eleven participants followed the instructions in Phase 2 and ten (five flexible and five inflexible) stopped following the instruction in Phase 3 . The results suggest that instruction-following tends to be abandoned when it produces reinforcer loss, independently of whether the participant is classified as flexible or inflexible. history

Keywords: Rules and contingencies, reinforcement loss, participants flexible and inflexible; pre-experimental

A maior parte dos autores concorda, no que se refere ao comportamento governado por regras, que este comportamento é estabelecido e mantido por uma história pré-experimental de reforço social para o seguir regras (Baron \& Galizio, 1983; Catania, Matthews \& Shimoff,
1990; Cerutti, 1989; Hayes, Brownstein, Zettle, Rosenfarb \& Korn, 1986; Joyce \& Chase, 1990; Malott, 1989; Otto, Torgrud \& Holborn, 1999; Skinner, 1969; Torgrud \& Holborn, 1990; Zettle \& Hayes, 1982). Consistente com essa visão, alguns estudos têm

Endereço para correspondência: Universidade Federal do Pará. Rua Augusto Corrêa, 01. Núcleo de Teoria e Pesquisa do Comportamento. CEP. 66075-110. Guamá. Belém, PA. Brasil. 88143030; e-mail: arpkeuffer@hotmail.com. Este trabalho foi baseado nos dados do Experimento 3 do projeto de doutorado do primeiro autor e realizado sob a orientaçāo do segundo e co-orientação do terceiro autor. O trabalho foi realizado com o auxílio do CNPq, em forma de bolsa de doutorado concedida ao primeiro autor, e de bolsa de produtividade em pesquisa, concedida ao segundo e ao terceiro autor. 
procurado investigar quais seriam os efeitos, sobre a manutenção do seguir regras, das consequências produzidas por este comportamento (N. M. A. Albuquerque, Paracampo \& L. C. Albuquerque, 2004; Galizio, 1979; Paracampo \& Albuquerque, 2004; Paracampo, Albuquerque, Farias, Carvalló \& Pinto, 2007) e de histórias préexperimentais de seguimento de regras (Pinto, Paracampo \& Albuquerque, 2006; Wulfert, Greenway, Farkas, Hayes \& Dougher, 1994).

Alguns resultados têm sugerido que a manutenção ou não do comportamento de seguir regras depende, em parte, do tipo de consequência produzida por esse comportamento (N. M. A. Albuquerque et al., 2004; Galizio, 1979; Paracampo \& Albuquerque, 2004; Paracampo et al., 2007). Mais especificamente, os resultados desses estudos têm sugerido que o comportamento de seguir regras discrepantes tem maior probabilidade de ser abandonado quando produz consequências aversivas (como a perda de reforços) do que quando produz outros tipos de consequências (como a não obtenção de reforços). Por exemplo, Paracampo e Albuquerque (2004) expuseram quatro crianças a um procedimento de escolha de acordo com o modelo. Em cada tentativa, um arranjo de estímulos constituído de um estímulo modelo e dois estímulos de comparação eram apresentados ao participante, que deveria apontar para um dos estímulos de comparação (diferente ou igual ao modelo) na presença de um estímulo contextual (luz vermelha ou verde). A Fase 1 da Condição 2 era iniciada com uma instrução que especificava as respostas que deveriam ser emitidas para evitar a perda de moedas. Nas Fases 1 e 3 nenhuma resposta produzia perda. Na Fase 2, a emissão das respostas descritas pela instrução na Fase 1 produzia a perda de moedas. Os resultados mostraram que, quando o seguimento de regra passou a produzir perda de moedas na Fase 2, todos os quatro participantes deixaram de seguir a regra.

Contudo, outros resultados têm sugerido que diferenças individuais constituem outro fator que pode estar correlacionado com a manutenção ou não do comportamento de seguir regras discrepantes das contingências programadas em situações experimentais (Wulfert et al., 1994). Para estes autores, a história de cada indivíduo pode contribuir para produzir indivíduos mais ou menos seguidores de regras que outros, isto é, mais ou menos inflexíveis que outros. Eles definiram inflexibilidade como o seguimento excessivo de regras, o que sugere que a manutenção do seguir regras discrepantes em laboratório pode corresponder à inflexibilidade do comportamento no dia a dia.

Em um dos experimentos conduzidos por Wulfert et al. (1994) para avaliar se a manutenção do seguir regras discrepantes das contingências programadas em situações experimentais está relacionada a diferenças individuais entre os participantes expostos a tais situações, foram formados quatro grupos com cinco participantes em cada. Cada participante foi exposto a duas sessões. $\mathrm{Na}$ Sessão 1, foram expostos a um esquema FR 8 e na Sessão 2 a um esquema DRL 4. Cada participante dos Grupos 1 e 2 foi exposto à instrução correspondente às contingências no início de cada uma das duas sessóes. Cada participante dos Grupos 3 e 4 foi exposto à instrução correspondente no início da Sessão 1 e à instrução discrepante das contingências no início da Sessão 2. Os participantes dos Grupos 1 e 3 foram classificados como inflexíveis e os 
dos Grupos 2 e 4 foram classificados como flexíveis com base em suas respostas a um questionário sobre inflexibilidade, isto é, com base em suas respostas à Escala de Rigidez desenvolvida por Rehfisch (1958) ${ }^{1}$. Na Sessão 1 todos os participantes seguiram a instrução correspondente. Na Sessão 2, os participantes dos Grupos 1 e 2 seguiram a instrução correspondente. Os participantes do Grupo 3 seguiram a instrução discrepante e três dos quatro participantes do Grupo 4, deixaram de seguir a instrução discrepante. Ou seja, os participantes inflexíveis (Grupo 3) tenderam a seguir a instrução discrepante, enquanto os participantes flexíveis (Grupo 4) tenderam a deixar de seguir a instrução. Wulfert e colaboradores propuseram que as diferenças de desempenho observadas entre os participantes inflexíveis e os participantes flexíveis estão relacionadas a diferentes histórias préexperimentais, que teriam gerado diferentes padrões de comportamento de seguir regras. Participantes inflexíveis teriam menor probabilidade de apresentar desempenho sensível às contingências programadas, devido a uma suposta história pré-experimental de punição por não seguir regras.

Nesta mesma linha de investigação, Pinto et al. (2006) procuraram avaliar se a manutenção, ou não, do seguir regras discrepantes das contingências de reforço programadas em situações experimentais depende mais da história experimental do ouvinte ou da sua história pré-experimental, inferida de suas respostas a um questionário sobre inflexibilidade. Participantes previamente classificados como flexíveis e como inflexíveis foram expostos a duas condiçôes experimentais que diferiam quanto à forma de estabelecimento da sequência correta na Sessão 2. Cada condição era constituída de quatro sessões. $\mathrm{Na}$ Condição 1 (Reforço diferencial), a Sessão 1 era iniciada com a apresentação de uma instrução mínima, as Sessões 2 e 3 com a apresentação de um arranjo de estímulos, e a Sessão 4 com a instrução discrepante das contingências. $\mathrm{Na}$ Condição 2 (Instrução), a Sessão 1 era iniciada com a instrução mínima, a Sessão 2 com a instrução correspondente às contingências, a Sessão 3 com um arranjo de estímulos e a Sessão 4 com a regra discrepante. Nas duas condiçôes, a Sessão 1 era constituída de 10 tentativas de linha de base onde nenhuma resposta era reforçada. As contingências de reforço em vigor na Sessão 2 eram alteradas na Sessão 3, e as contingências na Sessão 3, eram mantidas inalteradas na Sessão 4.

Os resultados mostraram que, na Condição 1 , todos os oito participantes mudaram seus desempenhos na Sessão 3, quando as contingências foram alteradas. $\mathrm{Na}$ Sessão 4, três dos quatro participantes inflexíveis seguiram a instrução discrepante e três dos quatro participantes flexíveis deixaram de seguir esta instrução. $\mathrm{Na}$ Condição 2, três dos quatro participantes flexíveis mudaram seus desempenhos na Sessão 3 e os quatro participantes inflexíveis continuaram seguindo a instrução correspondente nessa sessão. $\mathrm{Na}$ Sessão 4, os três participantes que na Sessão 3 haviam apresentado um desempenho sob controle das contingências, deixaram de seguir a instrução discrepante e os cinco participantes que na Sessão 3 haviam permanecido seguindo a instrução correspondente, continuaram respondendo incorretamente, seguindo a

\footnotetext{
${ }^{1}$ De acordo com Wulfert et al. (1994), esta escala parece apropriada porque ela tem validade reconhecida e foi empiricamente derivada. Além disso, ela descreve uma ampla variedade de situações que, quando respondidas de determinada forma, implicam em inibição, conservadorismo, intolerância, tendência a persistência e inflexibilidade.
} 
instrução discrepante em todas as tentativas da Sessão 4. Em síntese, os resultados mostraram que, independente da condição experimental, seis dos oito participantes flexíveis deixaram de seguir a instrução discrepante na Sessão 4; e sete dos oito participantes inflexíveis mantiveram o comportamento de seguir a instrução discrepante na Sessão 4. Estes resultados são similares aos resultados obtidos no estudo de Wulfert et al. (1994), que também mostrou que participantes inflexíveis tendem a seguir regras discrepantes; diferente de participantes flexíveis, que tendem a deixar de seguir tais regras. Tais resultados também sugerem que os autorrelatos indicativos de flexibilidade e os autorrelatos indicativos de inflexibilidade, identificados a partir da aplicação da Escala de Rigidez desenvolvida por Rehfisch (1958), podem ser utilizados para se prever a ocorrência da manutenção, ou não, do seguir regras discrepantes das contingências programadas em situações experimentais.

Considerando os resultados descritos anteriormente, não fica claro nos estudos que têm investigado os efeitos das consequências produzidas pelo comportamento controlado por regras na manutenção deste comportamento $(\mathrm{N}$. M. A. Albuquerque et al., 2004; Galizio, 1979; Paracampo \& Albuquerque, 2004; Paracampo et al., 2007), se o seguir a regra discrepante deixou de ocorrer porque produzia perda de reforçadores ou porque os participantes tinham uma história pré-experimental de reforço para o não seguir regras, isto é, porque eram flexíveis. Do mesmo modo, também não fica claro nos estudos que têm investigado os efeitos de histórias préexperimentais na manutenção do comportamento controlado por regras (Wulfert et al., 1994; Pinto et al., 2006), se o seguir a regra discrepante foi mantido, porque os participantes tinham uma história pré-experimental de reforço para o seguir e de punição para o não seguir regras (isto é, porque eles eram inflexíveis) ou porque o seguir regras não produzia perda de reforçadores.

Diante disso, o presente estudo pretendeu verificar se participantes previamente classificados de inflexíveis tem maior probabilidade de seguir regras discrepantes das contingências do que participantes previamente classificados de flexíveis, quando o seguir regras discrepantes produz perda de reforçadores. $\mathrm{O}$ procedimento de escolha de acordo com o modelo, empregado como tarefa experimental, foi adaptado do produzido por L. C. Albuquerque (1989).

\section{Método}

\section{Participantes}

Na primeira etapa deste estudo [exposição dos participantes a um questionário sobre inflexibilidade, isto é, à Escala de Rigidez desenvolvida por Rehfisch (1958)] foram convidados 272 estudantes universitários, sem história experimental prévia, de diversos cursos (exceto o de Psicologia), matriculados em diferentes semestres. Os participantes foram voluntários, convidados a participar do experimento por meio de um convite oral feito pelo experimentador em diferentes salas de aula de uma universidade. Desses 272, 11 participantes (seis com classificação indicativa de flexibilidade e cinco com classificação de inflexibilidade) foram convidados a participar da segunda etapa da pesquisa (exposição dos participantes às regras e às contingências programadas).

\section{Material e equipamentos}

Na primeira etapa do estudo foi utilizado um questionário, construído por Rehfisch 
(1958), denominado de Escala de Rigidez (o Anexo A apresenta uma tradução dessa escala, feita por Jonas, 2001), o qual é baseado no Minnesota Multiphasic Personality Inventory e no California Personality Inventory. A Escala de Rigidez constitui-se de um questionário com 39 itens do tipo verdadeiro / falso. Na segunda etapa do estudo foi utilizado um computador com um programa (software) desenvolvido em ambiente Windows especialmente para uso da pesquisa, de acordo com seus objetivos. $\mathrm{O}$ programa, uma versão informatizada do procedimento desenvolvido por Albuquerque (1989), foi produzido para apresentar as instruções, as contingências programadas e registrar as respostas dos participantes.

Durante o experimento, os estímulos apareciam na tela do computador. Acima e ao centro da tela, havia um contador que registrava os pontos. Abaixo do contador, havia uma caixa de texto onde eram apresentadas as instruções, bem como a consequência verbal que seguia cada sequência de resposta incorreta emitida pelo participante. As instruções foram gravadas em um pen drive e também eram apresentadas aos participantes por intermédio das caixas de som. Abaixo da caixa de texto era apresentado um arranjo de estímulos, constituído de um estímulo modelo (localizado na metade superior da tela) e de três estímulos de comparação (dispostos em fileira, um ao lado do outro, abaixo do estímulo modelo). Esses estímulos eram figuras geométricas variando em três dimensôes: forma (quadrado, círculo, retângulo e triângulo), cor (azul, vermelha e amarela) e espessura (grossa e fina). Estas figuras formavam diferentes arranjos de estímulos. Cada estímulo de comparação apresentava apenas uma dimensão - cor (C), espessura (E) ou forma (F) - em comum com o estímulo modelo e diferia nas demais. As respostas de escolha emitidas pelos participantes eram registradas automaticamente pelo programa. Os reforçadores eram pontos que eram trocados por dinheiro no final da pesquisa. Cada ponto valia $\mathrm{R} \$ 0.10$ (dez centavos de real).

\section{Procedimento}

Os participantes, previamente classificados de flexíveis e de inflexíveis na primeira etapa deste estudo, eram expostos a três fases experimentais na segunda etapa. A Fase 1 era iniciada com uma instrução mínima, a Fase 2, com uma instrução correspondente às contingências e a Fase 3, com uma instrução discrepante das contingências. Na Fase 1 (linha de base), nenhuma sequência era punida. $\mathrm{O}$ seguimento de instrução evitava a perda de reforçadores na Fase 2 e produzia a perda de reforçadores na Fase 3.

$\mathrm{Na}$ primeira etapa do estudo, o experimentador aplicou a Escala de Rigidez a 272 estudantes. Após o estudante responder ao questionário, o experimentador somava as respostas corretas (autorrelatos), de acordo com o gabarito apresentado no texto de Rehfisch (1958). Foram considerados autorrelatos indicativos de flexibilidade aqueles que se encontravam na faixa entre $0 \mathrm{e}$ 11 acertos (27\% ou menos do total de acertos possíveis) e foram considerados autorrelatos indicativos de inflexibilidade aqueles que se encontravam na faixa entre 29 e 39 acertos (75\% ou mais do total de acertos possíveis). Dos 272 participantes, apenas cinco apresentaram autorrelatos dentro da faixa indicativa de inflexibilidade e apenas seis apresentaram autorrelatos dentro da faixa indicativa de flexibilidade. Estes 11 participantes foram convidados a participar 
da segunda etapa do estudo. Todos os participantes desta etapa concordaram em participar do estudo assinando o Termo de Consentimento Livre e Esclarecido.

Durante as sessões experimentais, cada participante ficava sentado sozinho, frente à mesa com o computador, em uma sala reservada para o experimento, enquanto o experimentador aguardava do lado de fora da sala. Em cada tentativa, na presença de um arranjo de estímulos, o participante deveria tocar em cada um dos três estímulos de comparação em uma dada sequência. Caso a sequência de respostas emitida estivesse de acordo com as contingências programadas (sequência correta), não era retirado ponto no contador, também não era apresentada frase na caixa de texto, e um novo arranjo era apresentado automaticamente. Caso a sequência de respostas fosse incorreta, era retirado um ponto no contador, apresentada a frase "Você perdeu um ponto" e era apresentado um novo arranjo. Havia um intervalo de 2 segundos entre uma tentativa e outra.

$\mathrm{Na}$ primeira sessão, quando participante e experimentador entravam na sala, o computador estava sobre a mesa, ligado e visível ao participante. $\mathrm{O}$ experimentador pedia ao participante para sentar-se na cadeira e solicitava que lesse e assinasse o Termo de Consentimento Livre e Esclarecido desta etapa. Em seguida o experimentador confirmava o nome, idade, curso do mesmo. Então, o experimentador clicava com o mouse na tela para dar início ao programa e dizia que estaria do lado de fora da sala caso o participante precisasse. Na primeira tela era apresentado um arranjo de estímulos acompanhado das seguintes orientações escritas e do som correspondente:
"Este objeto que irá piscar aqui em cima é o modelo. Estes três objetos que irão piscar aqui em baixo são para você comparar com o modelo. Nós vamos chamar estes três objetos, aqui em baixo, de objetos de comparação. Observe que cada um destes três objetos de comparação tem uma única propriedade comum ao modelo. [Este que irá piscar tem a mesma espessura do modelo. Este que irá piscar agora tem a mesma cor do modelo. Este que irá piscar em seguida tem a mesma forma do modelo]. Inicialmente você receberá 160 pontos no contador, que serão trocados por dinheiro no final da pesquisa. Durante a pesquisa você poderá perder pontos. Quando você perder pontos, eles serão retirados desses 160 pontos e a frase "Você perdeu um ponto" aparecerá. Veja como um ponto é retirado no contador. (O programa automaticamente retirava um ponto no contador como demonstração). Quando você não perder pontos, nenhum ponto será retirado no contador e nenhuma frase aparecerá". Este procedimento era repetido mais uma vez e ocorria apenas no início da primeira sessão. $\mathrm{Na}$ segunda vez em que estas orientações preliminares eram apresentadas, o trecho entre colchetes era omitido.

Os 11 participantes, os cinco classificados como inflexíveis e os seis classificados como flexíveis, foram expostos a três fases, conforme indicado na Tabela 1. A Fase 1 era iniciada com a apresentação da seguinte instrução mínima: "Aponte em sequência para cada um dos três objetos de comparação". A Fase 1 era constituída de 10 tentativas de linha de base em relação a qual eram avaliados os efeitos da introdução da instrução correspondente às contingências na Fase 2. Durante a Fase 1 nenhuma resposta produzia perda ou ganho de pontos. 
A Fase 2 era programada para ser iniciada com a apresentação da seguinte instrução correspondente às contingências programadas:

"Quando aparecerem estes objetos na tela você deve fazer o seguinte: Primeiro toque no objeto de comparação que tem a mesma cor do objeto modelo. Depois toque no que tem a mesma espessura do objeto modelo. Em seguida toque no que tem a mesma forma do objeto modelo. Ou seja, você deve tocar primeiro na mesma cor, depois na mesma espessura e em seguida na mesma forma. Entendeu? Toque no que você deve fazer. Fazendo isso, você poderá evitar perder pontos. Cada ponto que você evitar perder será trocado por R $\$ 0,10$ (dez centavos de real), ao final da pesquisa”.

Contudo, se na Fase 1 o participante respondesse na sequência $\mathrm{CEF}$ em mais de 50\% das tentativas, a Fase 2 era iniciada com a instrução correspondente especificando a sequência CFE. Durante a Fase 2, o seguir a instrução correspondente, bem como a emissão de qualquer outra sequência, não produzia perda de pontos. Esta fase era encerrada após a ocorrência de 80 tentativas.

A Fase 3 era iniciada com a apresentação da seguinte instrução discrepante das contingências programadas:

"Quando aparecerem estes objetos na tela, você deve fazer o seguinte: Primeiro toque no objeto de comparação que tem a mesma forma do objeto modelo. Depois toque no que tem a mesma cor do objeto modelo. Em seguida toque no que tem a mesma espessura do objeto modelo. Ou seja, você deve tocar primeiro na mesma forma, depois na mesma cor e em seguida na mesma espessura. Entendeu? Toque no que você deve fazer. Fazendo isso, você poderá evitar perder pontos. Cada ponto que você evitar perder será trocado por $\mathrm{R} \$ 0,10$ (dez centavos de real), ao final da pesquisa”.

Durante a Fase 3, apenas o seguir a instrução discrepante produzia perda de pontos; a emissão de qualquer outra sequência não produzia perda de pontos. Esta fase era encerrada após a ocorrência de 80 tentativas.

A participação dos estudantes no experimento era encerrada depois de ser atingido o critério de encerramento da Fase 3.

\section{Resultados}

A Tabela 2 mostra o número de sequências de respostas emitidas pelos participantes flexíveis (PF1, PF2, PF3, PF4, PF5 e PF6) e inflexíveis (PI7, PI8, PI9, PI10 e PI11) durante a Fase 1 (linha de base). Os participantes apresentaram um desempenho variável e nenhum emitiu a sequência CEF em mais de $50 \%$ das tentativas. Assim, a sequência CEF

Tabela 1

Esquema do procedimento.

\begin{tabular}{cccc}
\hline & Fase 1 & Fase 2 & Fase 3 \\
\hline $\begin{array}{c}\text { Sequências de respostas instruídas } \\
\text { Sequência que produz perda de } \\
\begin{array}{c}\text { reforçador } \\
\text { Critério de encerramento }\end{array}\end{array}$ & nenhuma & CEF ou CFE & FCE \\
nenhuma & FCE & \\
\hline
\end{tabular}

Nota: $\mathrm{O}$ procedimento foi realizado com 11 participantes, seis classificados de flexíveis e cinco classificados de inflexíveis. $\mathrm{C}=$ resposta à dimensão cor. $\mathrm{E}=$ resposta à espessura. $\mathrm{F}$ = resposta à forma. 
foi selecionada para ser descrita pela instrução correspondente no inicio da Fase 2 para todos os participantes.

A Figura 1 mostra a frequência acumulada de sequências de respostas corretas (linha cheia) e incorretas (linha tracejada) emitidas pelos participantes flexíveis (PF) e inflexíveis (PI), durante as Fases 2 e 3. Pode-se observar que, na Fase 2, quando a instrução correspondente foi apresentada e o seguir essa instrução evitava a perda de pontos, todos os 11 participantes, independentemente de serem flexíveis ou inflexíveis, responderam corretamente, seguindo esta instrução. $\mathrm{Na}$ Fase 3, quando a instrução discrepante foi apresentada e o seguir essa instrução produzia a perda de pontos, todos os 11 participantes iniciaram seguindo a instrução discrepante, isto é, respondendo incorretamente. Posteriormente, cinco (PF1, PF2, PF3, PF4 e PF5) dos seis participantes flexíveis e todos os inflexíveis (PI7, PI8, PI9, PI10 e PI11) deixaram de seguir a instrução discrepante e passaram a responder corretamente, emitindo a sequência (CEF) previamente descrita pela instrução correspondente no início da Fase 2. O Participante PF6 (flexível) foi o único dos 11 participantes que seguiu a instrução discrepante durante a Fase 3, apesar de o comportamento de seguir esta instrução produzir a perda de pontos.

A Tabela 3 mostra os principais dados da Fase 3. Nota-se que os Participantes flexíveis PF1, PF2, PF3, PF4 e PF5, ao deixarem de seguir a instrução discrepante, passaram a responder corretamente na $4^{\mathrm{a}}, 7^{\mathrm{a}}, 4^{\mathrm{a}}, 2^{\mathrm{a}}$ e $4^{\mathrm{a}}$ tentativas, respectivamente. Já os Participantes inflexíveis PI7, PI8, PI9 PI10 e PI11 deixaram de seguir a instrução discrepante e passaram a responder corretamente na $3^{\mathrm{a}}, 28^{\mathrm{a}}, 20^{\mathrm{a}}, 5^{\mathrm{a}}$ e $4^{\mathrm{a}}$ tentativas, respectivamente. Observa-se também que entre os participantes que abandonaram o seguir a instrução discrepante, PI8 e PI9 foram os que seguiram a instrução discrepante durante um maior número de tentativas.

A Tabela 4 mostra as respostas dos participantes a alguns dos itens do questionário. Estes itens foram selecionados apenas para ilustração. Nota-se algumas diferenças entre os relatos dos participantes inflexíveis e flexíveis.

Tabela 2

Número de sequência de respostas emitidas por cada participante durante a Fase 1 (linha de base).

\begin{tabular}{ccccccc}
\hline Participantes & CEF & CFE & FCE & FEC & ECF & EFC \\
\hline PF1 & 03 & 00 & 00 & 02 & 04 & 00 \\
PF2 & 01 & 01 & 01 & 02 & 04 & 00 \\
PF3 & 01 & 01 & 03 & 01 & 01 & 02 \\
PF4 & 00 & 05 & 01 & 02 & 00 & 01 \\
PF5 & 02 & 00 & 02 & 02 & 00 & 03 \\
PF6 & 00 & 02 & 06 & 00 & 00 & 01 \\
PI7 & 02 & 01 & 02 & 00 & 02 & 02 \\
PI8 & 01 & 01 & 03 & 00 & 01 & 03 \\
PI9 & 00 & 04 & 01 & 00 & 01 & 03 \\
PI10 & 02 & 01 & 01 & 02 & 01 & 02 \\
PI11 & 03 & 00 & 03 & 01 & 01 & 01 \\
\hline
\end{tabular}

Nota: $\mathrm{C}$ = resposta à dimensão cor. $\mathrm{E}$ = resposta à espessura. $\mathrm{F}$ = resposta à forma. $\mathrm{PF}=$ Participante flexível. $\mathrm{PI}$ = Participante inflexível. 


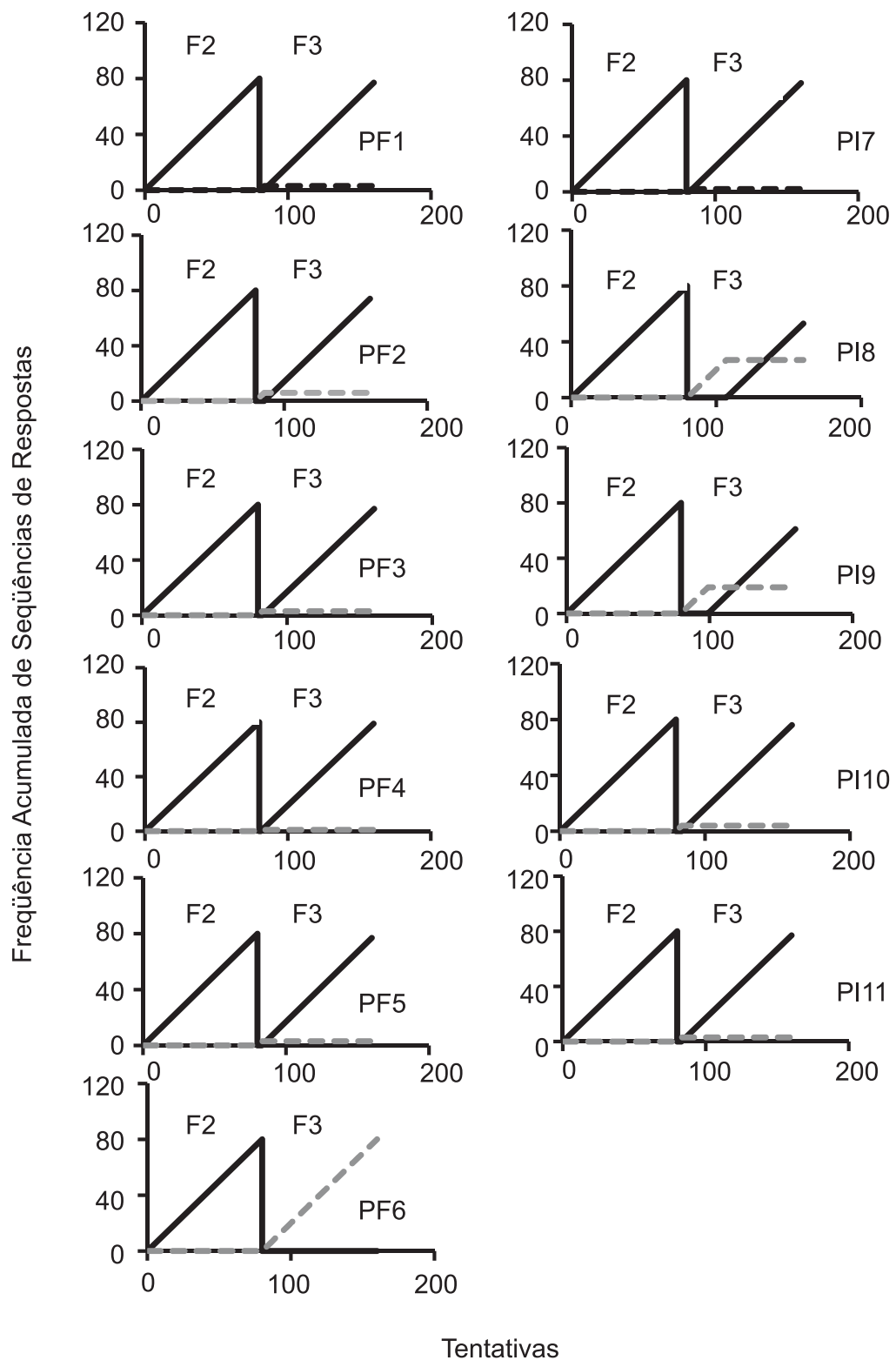

Figura 1. Frequência acumulada de sequências de respostas emitidas por cada particIpante flexível (PF) e inflexível (PI), nas Fases (F) 2 e 3 do experimento. Linha cheia indica sequência correta e linha tracejada indica sequência incorreta.

Por exemplo, no Item 9, quatro dos cinco participantes inflexíveis e apenas dois dos seis participantes flexíveis relataram que era verdadeira a afirmação: "Eu sempre sigo a regra: negócios antes do prazer”. No Item 13, também quatro dos cinco participantes inflexíveis e apenas dois dos seis participantes flexíveis relataram que era verdadeira a afirmação: "Eu considero que um modo de vida bem organizado, com horários regulares é o ideal para meu temperamento”.

\section{DISCUSSÃo}

Os resultados do presente estudo replicam os resultados obtidos em estudos que têm 


\section{Tabela 3}

Número ordinal da tentativa a partir da qual o seguir a instrução discrepante das contingências deixou de ocorrer na Fase 3.

\begin{tabular}{cc}
\hline Participantes & Tentativa \\
\hline PF1 & $4^{\mathrm{a}}$ \\
PF2 & $7^{\mathrm{a}}$ \\
PF3 & $4^{\mathrm{a}}$ \\
PF4 & $2^{\mathrm{a}}$ \\
PF5 & $4^{\mathrm{a}}$ \\
PF6 & Não \\
PI7 & $3^{\mathrm{a}}$ \\
PI8 & $28^{\mathrm{a}}$ \\
PI9 & $20^{\mathrm{a}}$ \\
PI10 & $5^{\mathrm{a}}$ \\
PI11 & $4^{\mathrm{a}}$ \\
\hline
\end{tabular}

Nota: Todos os 11 participantes seguiram a instrução discrepante na primeira tentativa da Fase 3. "Não", indica que o seguir a instrução discrepante não deixou de ocorrer definitivamente, isto é, indica que ele foi mantido durante a maior parte da fase (caso de P6I). PF = Participantes flexíveis. PI = Participantes inflexíveis.

investigado os efeitos de consequências produzidas pelo comportamento controlado por regras na manutenção deste comportamento (N. M. A. Albuquerque et al., 2004; Galizio, 1979; Paracampo \& Albuquerque, 2004; Paracampo et al., 2007). Mas em adição, os resultados do presente estudo também mostram que o seguir regras tende a ser abandonado quando produz perda de reforçadores, independentemente de se o participante é classificado de flexível ou de inflexível.

Os resultados do presente estudo, juntos com os resultados dos estudos que têm investigado os efeitos de histórias préexperimentais (inferidos de autorrelatos indicativos de padrões de comportamentos flexíveis e inflexíveis) sobre a manutenção do seguir regras (Pinto et al., 2006; Wulfert et al., 1994), também apóiam a sugestão de que os eventuais efeitos de histórias pré-experimentais dependem de condições experimentais atuais (Albuquerque, de Souza, Matos \& Paracampo, 2003; Oliveira \& Albuquerque, 2007). Assim, pode-se sugerir que as histórias préexperimentais de seguir regras teriam maior probabilidade de interferir na manutenção do seguir regras discrepantes das contingências, quando a combinação das condições que favorecem e que não favorecem o seguir e o não seguir regra fosse equilibrada (como ocorreu no estudo de Pinto et al., 2006) do que quando aquela combinação favorecesse mais o não seguir regra (como ocorreu no presente estudo).

No estudo de Pinto et al. (2006), as duas condiçôes a que os participantes foram expostos favoreciam tanto o seguir quanto o não seguir a regra discrepante das contingências. Favoreciam o seguir a regra discrepante na medida em que o experimentador ficava presente na situação experimental, monitorando o seguir essa regra, uma vez que ele consequenciava diferencialmente este comportamento. Favoreciam o não seguir a regra discrepante na medida em que o seguir essa regra não produzia a consequência reforçadora por ela especificada; ao invés, produzia a não obtenção de ponto. Mas a Condição 1 favorecia mais o não seguir a regra discrepante do que a Condição 2. $\mathrm{Na}$ Condição 1, o comportamento alternativo ao especificado pela regra discrepante havia sido estabelecido por reforço diferencial na Sessão 2 e este fato deve ter contribuído para que todos os oito participantes dessa condição tivessem apresentado um desempenho sensível à mudança nas contingências na Sessão 3. Em outros estudos (Albuquerque \& Silva, 2006; Silva \& Albuquerque, 2006; Silva \& Albuquerque, 2007), que utilizaram um procedimento similar ao usado no estudo de 
Tabela 4

Autorrelatos dos participantes flexíveis (PF) e inflexíveis (PI) diante de alguns dos itens do questionário.

\begin{tabular}{cccccccccccc}
\hline $\begin{array}{c}\text { Itens do } \\
\text { questionário }\end{array}$ & PF1 & PF2 & PF3 & PF4 & PF5 & PF6 & PI7 & PI8 & PI9 & PI10 & PI11 \\
\hline 7 & F & F & F & F & V & V & V & V & V & V & V \\
9 & F & F & V & F & F & V & V & F & V & V & V \\
13 & F & F & F & F & V & V & V & V & V & V & F \\
16 & V & V & V & V & F & F & F & F & V & V & V \\
17 & F & F & F & V & F & V & V & V & V & V & V \\
18 & F & F & V & F & F & V & V & V & V & V & V \\
25 & F & F & F & V & F & V & V & V & V & V & F \\
27 & F & F & F & F & F & V & V & V & V & F & V \\
34 & F & F & F & F & V & V & V & V & V & V & V \\
36 & V & V & F & F & F & V & V & V & V & V & V \\
\hline
\end{tabular}

Nota: $\mathrm{V}=$ Resposta do tipo verdadeiro; F= Resposta do tipo falso (F); 7 = Eu não gosto de coisas que sejam incertas ou imprevisíveis; 9 = Eu sempre sigo a regra: negócios antes do prazer; 13 = Eu considero que um modo de vida bem organizado, com horários regulares é o ideal para o meu temperamento; 16 $=$ Eu às vezes sinto que posso mudar minha opinião com muita facilidade; 17 = Eu não gosto de me envolver em nenhum projeto a menos que tenha uma boa idéia de como ele vai terminar; 18 = Eu acho difícil por de lado uma tarefa que comecei, nem que seja por pouco tempo; 25 = Me aborrece quando algo inesperado interrompe minha rotina diária; 27 = Eu me sentiria desconfortável vestindo qualquer outra coisa que não uma roupa convencional; 34 = Eu devo admitir que tento ver o que os outros pensam antes de fazer uma escolha; 36 = Eu devo admitir que fico zangado quando outras pessoas interferem em minhas atividades diárias.

Pinto et al. (2006), os participantes (estudantes universitários que não haviam sido previamente classificados de flexíveis ou inflexíveis) que apresentaram um desempenho sensível à mudança nas contingências na Sessão 3, tenderam a abandonar o seguir a regra discrepante na Sessão 4. Diferente desses resultados, no estudo de Pinto et al., três dos quatro participantes inflexíveis seguiram a regra discrepante na Sessão 4 da Condição 1 . O que chama a atenção nesse resultado é o fato de estes participantes terem seguido a regra discrepante na Sessão 4, apesar de terem sido expostos a uma história na Sessão 3 em que o comportamento de mudar havia sido reforçado.

Mas, diferente das condições a que esses três participantes do estudo de Pinto et al. (2006) foram expostos, no presente estudo, os participantes foram expostos a condições que favoreciam ainda mais o não seguir a regra discrepante. Diferente do estudo de Pinto et al., no presente estudo, o experimentador não monitorava o seguir regra, uma vez que ele ficava do lado de fora da sala experimental. Também diferente do estudo de Pinto et al., no presente estudo, o seguir a regra discrepante era punido, uma vez que produzia a retirada de ponto. Além disso, no caso dos Participantes PI8 e PI9, o tempo de exposição à discrepância entre a regra e as contingências programadas (Bernstein, 1988) também deve ser considerado como mais uma condição que favoreceu o abandono do seguir a regra discrepante.

Deste modo, pode-se dizer que as condições experimentais no presente estudo, podem ter reduzido fortemente a probabilidade de que os participantes inflexíveis tivessem persistido seguindo a regra discrepante. Em 
outras palavras os participantes inflexíveis iniciaram a Fase 3 seguindo a regra discrepante, possivelmente porque tinham uma história préexperimental de seguir regras (Baron \& Galizio, 1983; Cerutti, 1989; Hayes et. al., 1986; Joyce \& Chase, 1990; Malott, 1989; Otto et. al., 1999; Skinner, 1969; Torgrud \& Holborn, 1990; Wulfert et al., 1994; Zettle \& Hayes, 1982). Mas não continuaram seguindo a regra discrepante, porque o seguir essa regra não produzia aprovação social do experimentador. Ao contrário, produzia punição.

Um problema maior, no entanto, consiste em explicar os dados do Participante PF6, que seguiu a regra discrepante. Os dados desse participante não são prontamente explicados pela proposição de que a manutenção ou não do comportamento de seguir regras depende, em parte, do tipo de consequência produzida por esse comportamento, uma vez que por essa proposição o seguir regras discrepantes teria maior probabilidade de ser abandonado quando produzisse perda de reforços (N. M. A. Albuquerque et al., 2004; Galizio, 1979; Paracampo \&Albuquerque, 2004; Paracampo et al., 2007). Também não são prontamente explicados pela proposição de que participantes flexíveis tenderiam a deixar de seguir regras discrepantes (Wulfert et al., 1994). Uma possibilidade seria supor, no caso específico desse participante, que a história experimental de reforço para o seguir a regra correspondente, construída na Fase 2, tenha contribuído para manter o seguir a regra discrepante na Fase 3 (Albuquerque, 1998; Albuquerque et al., 2003; Martinez \& Tomayo, 2005; Skinner, 1969, 1974). Essa história, construída na Fase 2, também pode ter contribuído para que os Participantes PI8 e PI9 tivessem seguido a instrução discrepante durante as primeiras tentativas da Fase 3.
Mas, por outro lado, os resultados do presente estudo também apóiam a sugestão de que o controle exercido por uma história experimental de reforço para o seguir regra correspondente pode substituir o controle exercido por uma regra discrepante das contingências sobre o comportamento por ela especificado (Albuquerque, 1998; Albuquerque et al., 2003; Oliveira \& Albuquerque, 2007). Isso pode ser afirmado uma vez que os Participantes PF1, PF2, PF3, PF4, PF5, PI7, PI8, PI9, PI10 e PI11, ao deixarem de seguir a regra discrepante na Fase 3, ao invés de variarem os seus desempenhos, passaram a emitir a sequência (CEF) previamente especificada pela regra correspondente e que havia sido reforçada na Fase 2. Pode-se sugerir, então, que o controle pela história de reforço para o seguir a regra correspondente, construída na Fase 2, contribuiu tanto para manter o seguir regras discrepantes, quanto para substituir o controle subsequente pela regra discrepante. Ou seja, pode-se sugerir que a história da Fase 2 também contribuiu para determinar os desempenhos na Fase 3.

Esta análise está de acordo com a proposição de que a manutenção do seguir regras depende da combinação entre os fatores que favorecem e os que não favorecem o seguir e o não seguir regras (Albuquerque et. al., 2003). Pesquisas futuras, que continuassem buscando identificar as condiçôes sob as quais histórias experimentais e histórias pré-experimentais teriam maior ou menor probabilidade de exercerem os seus efeitos poderiam contribuir para esclarecer qual seria o papel dessas histórias entre os fatores que favorecem e os que não favorecem a manutenção do comportamento de seguir e o de não seguir regras.

Em síntese, os resultados dos estudos de Pinto et al. (2006) e Wulfert et al. (1994), 
sugerem que os autorrelatos indicativos de flexibilidade e os autorrelatos indicativos de inflexibilidade, identificados a partir da aplicação da Escala de Rigidez desenvolvida por Rehfisch (1958), podem ser utilizados para se prever a manutenção do seguir regras discrepantes das contingências programadas em situaçôes experimentais. Diferente dos resultados desses dois estudos (Pinto e colaboradores e Wulfert e colaboradores), os resultados do presente estudo mostram que os autorrelatos indicativos de flexibilidade e os autorrelatos indicativos de inflexibilidade, por si só, não são suficientes para se prever a manutenção do seguir regras discrepantes. Os resultados do presente estudo, juntos com os obtidos nos estudos de Pinto e colaboradores e Wulfert e colaboradores, sugerem que, para tanto, também é necessário considerar as condições atuais em que o seguir regras ocorre. Além disso, os resultados do presente estudo sugerem que se pode inferir, a partir dos autorrelatos dos participantes, como os mostrados na Tabela 4, que participantes flexíveis e inflexíveis tem diferentes histórias préexperimentais de seguir regras. Mas tais autorrelatos não indicam o tipo específico de história de seguir regras de cada participante, isto é, não se pode identificar, apenas com base em tais autorrelatos, se a história préexperimental do participante era de reforço social para o seguir regras similares (Skinner, 1969, 1974), ou de reforço social em algumas situações que se generalizou para outras situações (Catania, 1998), ou de punição social do não seguir regras (Wulfert et al.,1994), ou de promessas de reforço para o seguir regras e ameaças de punição para o não seguir regras (Albuquerque, 2005). Contudo, independentemente do tipo história pré- experimental de seguir regras que cada participante trouxe para o laboratório, os resultados do presente estudo, juntos com os obtidos nos estudos de Pinto e colaboradores e Wulfert e colaboradores, sugerem que os eventuais efeitos de tal história dependem de variáveis atuais.

\section{REFERÊNCIAS}

Albuquerque, L. C. (1989). Efeitos de regras no controle do comportamento de escolha Resumos da XIX Reunião Anual de Psicologia da Sociedade de Psicologia de Ribeirão Preto, pp. 422-423.

Albuquerque, L. C. (1998). Efeitos de histórias experimentais sobre o seguimento subseqüentes de regras. Tese de Doutorado não-publicada. Instituto de Psicologia, Universidade de São Paulo. São Paulo: USP.

Albuquerque, L. C. (2005). Regras como instrumento de análise do comportamento. Em L. C. Albuquerque (Org.), Estudos do comportamento (pp.143-176). Belém: Edufpa.

Albuquerque, L. C., de Souza, D. G., Matos, M. A., \& Paracampo, C. C. P. (2003). Análise dos efeitos de histórias experimentais sobre o seguimento subseqüente de regras. Acta Comportamentalia, 11, 87-126.

Albuquerque, L. C., \& Silva, F. M. (2006). Efeitos da exposição a mudanças nas contingências sobre o seguir regras. Psicologia: Teoria e Pesquisa, 22, 101112.

Albuquerque, N. M. A., Paracampo, C. C. P., \& Albuquerque, L. C. (2004). Análise do papel de variáveis sociais e de conseqüências programadas no seguimento de instruçôes. Psicologia, Reflexão e Critica, 17, 31-42.

Baron, A., \& Galizio, M. (1983). Instructional control of human operant behavior. The Psychological Record, 33, 495-520. 
Bernstein, D. J. (1988). Laboratory lore and research practices in the experimental analysis of human behavior: Designing session logistics-how long, how often, how many? The Behavior Analyst, 11, 5158.

Catania, A. C. (1998). Learning. New Jersey: Prentice Hall.

Catania, A. C., Matthews, A., \& Shimoff, E. (1990). Properties of rule-governed behaviour and their implications. Em D. E. Blackman \& H. Lejeune (Orgs.), Behaviour analysis in theory and practice: Contributions and controversies (pp.215-230). Brighton: Lawrence Erlbaum.

Cerutti, D. T. (1989). Discrimination theory of rulegoverned behavior. Journal of the Experimental Analysis of Behavior, 51, 259-276.

Galizio, M. (1979). Contingency-shaped and rulegoverned behavior: Instructional control of human loss avoidance. Journal of the Experimental Analysis of Behavior, 31, 53-70.

Hayes, S. C., Brownstein, A. J., Zettle, R. D., Rosenfarb, I., \& Korn, Z. (1986). Rule governed behavior and sensitivity to changing consequences of responding. Journal of the Experimental Analysis of Behavior, 45, 237-256.

Jonas, A (2001). Efeitos de instruções sobre o desempenho em matching to sample e sua relaçāo com padrōes de sensibilidade comportamental a contingências. Tese de doutorado não-publicada, Instituto de Psicologia, Universidade de São Paulo. São Paulo, SP.

Joyce, J. H., \& Chase, P. N. (1990). Effects of response variability on the sensivity of rule-governed behavior. Journal of the Experimental Analysis of Behavior, 54, 251-262.

Malott, R. M. (1989). Achievement of evasive goals. Em S. C. Hayes (Org.), Rule governed behavior: Cognition, contingencies, and instructional control. (pp. 153-190). New York: Plenum.

Martinez, H. \& Tomayo, R. (2005). Interactions of contingencies, instructional accuracy, and instructional history in conditional discrimination. The Psychological Record, 55, 633-646.

Oliveira, V. L., \& Albuquerque, L. C. (2007). Efeitos de histórias experimentais e de esquemas de reforço sobre o seguir regras. Psicologia: Teoria e Pesquisa, 23, 217-228.

Otto, T. L., Torgrud, L. J., \& Holborn, S. W. (1999). An operant blocking interpretation of instructed insensitivity to schedule contingencies. The Psychological Record, 49, 663-684.

Paracampo, C. C. P., \& Albuquerque, L. C. (2004). Análise do papel das conseqüências programadas no seguimento de regras. Interação em Psicologia, 8, 237-245.

Paracampo, C. C. P., Albuquerque, L. C., Farias, A. F., Carvalló, B. N., \& Pinto, A. R. (2007). Efeitos de conseqüências programadas sobre o comportamento de seguir regras. Interação em Psicologia, 11, 161173.

Pinto, A. R., Paracampo, C. C. P., \& Albuquerque, L. C. (2006). Análise do controle por regras em participantes classificados de flexíveis e de inflexíveis. Acta Comportamentalia, 14, 171-194.

Rehfisch, J. M. (1958). A Scale for Personality rigidity. Journal of Consulting Psychology, 1, 11-15.

Silva, F. M., \& Albuquerque, L. C. (2006). Efeitos de perguntas e de histórias experimentais sobre o seguir regras. Psicologia: Teoria e Pesquisa, 22, 133-142.

Silva, L. S., \& Albuquerque, L. C. (2007). Efeitos de histórias comportamentais sobre o comportamento de seguir regras discrepantes das contingências. Interação em Psicologia, 11, 11-25.

Skinner, B. F. (1969). Contingencies of reinforcement: $A$ theoretical analysis. New York: Appleton-CenturyCrofts.

Skinner, B. F. (1974). About behaviorism. New York: Alfred A. Knopf.

Torgrud, L. J., \& Holborn, S. W. (1990). The effects of verbal performance descriptions on nonverbal operant responding. Journal of the Experimental 
Analysis of Behavior, 54, 273-291.

Wulfert, E., Greenway, D. E., Farkas, P., Hayes, E. C., \& Dougher, M. J. (1994). Correlation between selfreported rigidity and rule-governed insensitivity to operant contingencies. Journal of Applied Behavior Analysis, 27, 659-671.

Zettle, R. D., \& Hayes, S. C. (1982). Rule-governed behavior : A potential theoretical framework for cognitive-behavior therapy. Em P. C. Kendall (Org.), Advances in cognitive-behavioral research and therapy (pp. 73-118). New York: Academic Press.

Submetido em 07 de janeiro de 2009 Aceito em 12 de julho de 2009 